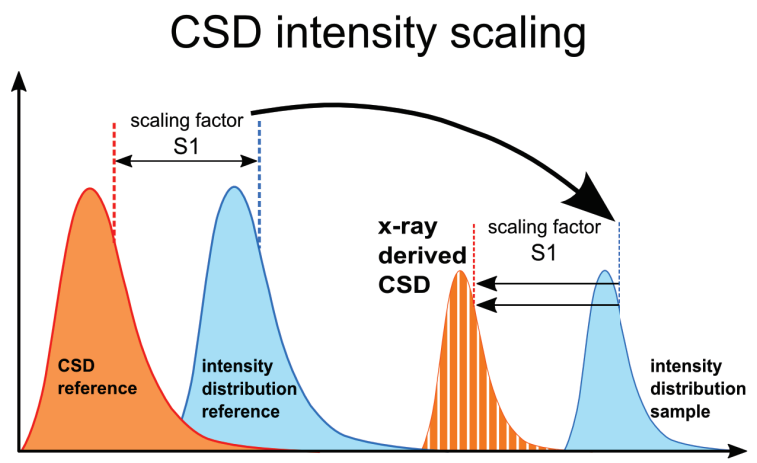

Figure 1. CSD intensity scaling: The scaling factor, S1, between the measured Intensity Distribution (ID) and the volume distribution (CSD) of the reference is used to derive the sample CSD from the measured sample ID.

Keywords: CSD, X-Ray, Crystallite Size Distribution
MS13-P4 Is it possible to collect reliable data on a laboratory diffractometer for a single crystal containing giant disordered supramolecules?

Eugenia V. Peresypkina ${ }^{1,2}$, Alexander V. Virovets ${ }^{1,2}$

1. University of Regensburg, Regensburg, Germany

2. Nikolaev Institute of Inorganic Chemistry SB RAS, Novosibirsk, Russia

email: peresyp@niic.nsc.ru

The advantages of the synchrotron radiation sources in diffraction studies are well-known. But laboratory diffractometers equipped with modern micro-focus X-ray tubes have their advantages, first of all, full-time availability that provides structure determination keeping pace with the ongoing chemical study, and enough space for laboratory equipment to manipulate samples. During last decade we systematically investigate structural chemistry of the compounds based on pentaphosphaferrocene, $\left[\mathrm{Cp}^{\mathrm{R}} \mathrm{Fe}\left(\eta^{5}-\mathrm{P}_{5}\right)\right] \quad\left(\mathrm{Cp}^{\mathrm{R}}=\eta^{5}-\mathrm{C}_{5} \mathrm{R}_{5}\right.$, $\left.\mathrm{R}=\mathrm{Me}, \mathrm{CH}_{2} \mathrm{Ph}\right)$, and $\mathrm{Cu}^{+}, \mathrm{Ag}^{+}\left(\mathrm{M}^{+}\right)$salts. Coordination of $\mathrm{M}^{+}$to $\mathrm{P}$ atoms of cyclo- $\mathrm{P}_{5}$ rings results in various giant supramolecules containing dozens of heavy atoms confined by $\mathrm{Cp}^{\mathrm{R}}$ ligands (Fig. 1) [1,2]. At that, inorganic core is often disordered resulting in weak high-angle reflections. The crystals usually demonstrate strong tendency for twinning and quick loosing of solvent and, therefore, cannot be pre-selected in the lab before transporting to the synchrotron.

Our equipment allows us to keep a sample in the argon atmosphere and select the crystals at about $-100^{\circ} \mathrm{C}$. Using Agilent Technologies SuperNova diffractometer $(\mathrm{CuK} \alpha$ micro-focus source, Titan ${ }^{\mathrm{S}} \mathrm{CCD}$ detector) and single crystals with $\mathrm{V} \sim 30000 \AA^{3}$ we systematically investigate how to measure weak intensities avoiding reflections overlap and how to improve the quality of diffraction data, the reliability of the structural model and quality factors by optimization of such factors as scan width, crystal-to-detector distance and detector mode, exposure time and redundancy. We use the results to tune the data collection strategies originally designed for small molecules and succeeded, for example, in the refinement of the crystal structures with following characteristics:

1: $\mathrm{a}=61.88, \mathrm{~b}=68.87, \mathrm{c}=69.66 \AA, \mathrm{V}=296829 \AA^{3}, F d d d$, 370 unique non-H positions, $\mathrm{d}>0.83 \AA, \mathrm{R} 1=0.15$

2: $a=43.12, b=42.13, c=43.28 \AA, \beta=92.31^{\circ}, V=78555$ $\AA^{3}, C 2 / c, 585$ non-H's, d $>0.83 \AA$, R $1=0.08$

3: $a=46.4, b=31.92, c=91.33 \AA, \beta=97.44^{\circ}, V=$ $134142 \AA^{3}, C 2 / c, 762$ non-H's, d>0.98 $\mathrm{A}, \mathrm{R} 1=0.13$

[1] E. V. Peresypkina et al (2014), Z. Kristallogr., 229, 735 , and references therein.

[2] F. Dielmann et al (2015), Chem.-A Eur. J., DOI: 10.1002/chem.201500692. 


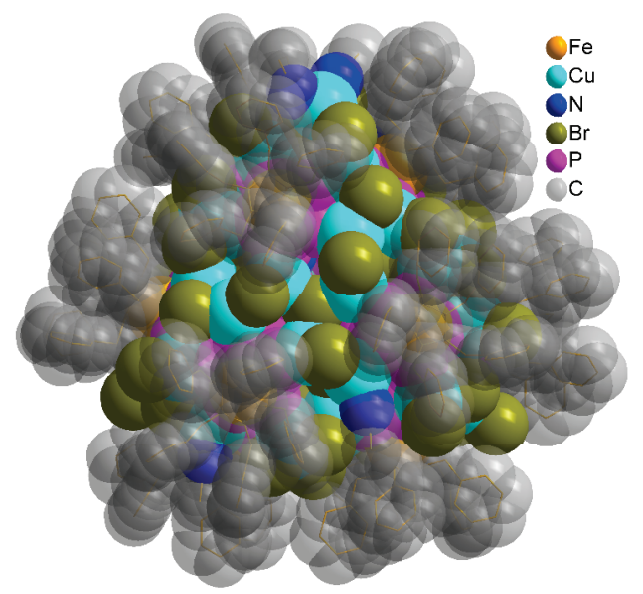

Figure 1. Supramolecule $\left[\left\{\mathrm{Cp}^{\mathrm{Bn}} \mathrm{Fe}\left(\eta^{5}-\mathrm{P}_{5}\right)\right\}_{12}\{\mathrm{CuBr}\}_{51}\{\mathrm{MeCN}\}_{8}\right]$ in $1, \mathrm{H}$ atoms are omitted for clarity.

Keywords: X-ray single-crystal diffraction, data collection strategies, model refinement, disorder, unstable crystals

\section{MS13-P5 Automated data analysis for X-ray diffraction experiments in chemical crystallography}

Markus Gerstel $^{1}$, David R. Allan ${ }^{1}$, Alun W. Ashton ${ }^{1}$, Paul V. Hathaway $^{1}$, Harriott Nowell ${ }^{1}$, Sarah A. Barnett ${ }^{1}$, Mark R. Warren ${ }^{1}$, Graeme Winter

1. Diamond Light Source, Harwell Science and Innovation Campus, Didcot, Oxfordshire OX11 0DE, England

email: markus.gerstel@ diamond.ac.uk

In chemical crystallography the routine use of fully automated data reduction (Winter \& McAuley, 2011) is not as widespread as in the closely related field of macromolecular crystallography (MX). Data processing times are not crucial for data collection on laboratory sources and did not constitute the rate-limiting step for collections at the two dedicated small-molecule synchrotron beamlines worldwide. As data collection times decrease through advances in detector technology, processing times become more important.

Here we present the current progress in adapting and extending MX data analysis and reduction software to chemical crystallography applications, and implementing these in an automated data processing pipeline at the dedicated chemical crystallography beamline I19 at the Diamond Light Source (Nowell et al., 2012). The beamline will, following an extensive hardware and software upgrade later this year, be equipped with a Pilatus 2M pixel-array detector. The concomitant reduction in data collection time and the expected increase in the volume of data collected necessitates a greater level of automation in terms of data processing and reduction. To achieve this goal MX software, such as GDA (General Data Acquisition framework; Gibbons et al., 2011) used for data collection at DLS, the data reduction expert system xia2 (Winter, 2010) and the Diffraction Integration for Advanced Light Sources (DIALS) software suite, are modified and extended to accommodate chemical crystallography requirements.

In chemical crystallography data are commonly collected using spherical or hemispherical data collection strategies, which are comprised of multiple sweeps based on the rotation method. In the proposed setup the auto-indexing solutions are continually refined throughout data acquisition, and, based on the relevant current indexing solution and hitherto collected data, further data collection strategies are evaluated using DIALS, and subsequently proposed to the experimenter.

Diffraction data are processed in the background using xia2 and DIALS, which both run as modules in the Computational Crystallography Toolbox (cctbx; Grosse-Kunstleve et al., 2002).

Gibbons, E.P., et al., Proceedings of the ICALEPCS (2011). 529-532.

Grosse-Kunstleve, Ralf W., et al., J. Appl. Cryst. (2002). 35.1, 126-136.

Nowell, H., et al., J. Synchrotron Rad. (2012). 19, 435-441.

Winter, G., J. Appl. Cryst. (2010). 43, 186-190.

Winter, G. and McAuley, K.E., Methods (2011). 55, 81-93.

Keywords: chemical crystallography, data reduction, automation 